\title{
Effects of dispersal and environmental heterogeneity on the replacement and nestedness components of $\beta$-diversity
}

\author{
Andros T. Gianuca, ${ }^{1,3}$ Steven A. J. Declerck, ${ }^{2}$ Pieter Lemmens, ${ }^{1}$ and Luc De Meester 1 \\ ${ }^{1}$ Laboratory of Aquatic Ecology, Evolution and Conservation, KU Leuven, Charles Deberiostraat 32, B 3000, Leuven, Belgium \\ ${ }^{2}$ Department of Aquatic Ecology, Netherlands Institute of Ecology (NIOO-KNAW), P.O. Box 50, 6700AB, Wageningen, \\ The Netherlands
}

\begin{abstract}
Traditionally metacommunity studies have quantified the relative importance of dispersal and environmental processes on observed $\beta$-diversity. Separating $\beta$-diversity into its replacement and nestedness components and linking such patterns to metacommunity drivers can provide richer insights into biodiversity organization across spatial scales. It is often very difficult to measure actual dispersal rates in the field and to define the boundaries of natural metacommunities. To overcome those limitations, we revisited an experimental metacommunity dataset to test the independent and interacting effects of environmental heterogeneity and dispersal on each component of $\beta$-diversity. We show that the balance between the replacement and nestedness components of $\beta$-diversity resulting from eutrophication changes completely depending on dispersal rates. Nutrient enrichment negatively affected local zooplankton diversity and generated a pattern of $\beta$-diversity derived from nestedness in unconnected, environmentally heterogeneous landscapes. Increasing dispersal erased the pattern of nestedness, whereas the replacement component gained importance. In environmentally homogeneous metacommunities, dispersal limitation created community dissimilarity via species replacement whereas the nestedness component remained low and unchanged across dispersal levels. Our study provides novel insights into how environmental heterogeneity and dispersal interact and shape metacommunity structure.
\end{abstract}

Key words: eutrophication; fragmentation; landscape connectivity; metacommunity; turnover; zooplankton.

\section{INTRODUCTION}

Biodiversity is a multifaceted concept that requires a multiscale approach to be fully understood (Segre et al. 2014). In addition to local scale diversity ( $\alpha$-diversity), differentiation among habitats ( $\beta$-diversity) is an important determinant of regional diversity. $\beta$-diversity, here defined as the dissimilarity in species composition among a pair of sites, can be partitioned into two components: dissimilarity derived from species replacement and dissimilarity derived from nestedness (Baselga 2010, Legendre 2014). The replacement component reflects changes in species identities (or relative abundances) between sites, whereas the nestedness component reflects to what extent the species-poor site contains a proper subset of the species-richer site (Baselga 2012, Legendre 2014). The replacement and nestedness components can contribute jointly to total dissimilarity among communities, but their relative importance will change depending on the ecological processes structuring metacommunities (Brendonck et al. 2015, Tonkin et al. 2016). Therefore, partitioning $\beta$-diversity into its replacement and nestedness components and linking such patterns to metacommunity drivers can provide additional insights into

Manuscript received 27 May 2016; revised 6 November 2016; accepted 16 November 2016. Corresponding Editor: Carla E. Cáceres.

${ }^{3}$ E-mail: andros.taroucogianuca@bio.kuleuven.be the mechanisms that shape biodiversity patterns across spatial scales (Hortal et al. 2011, Leprieur et al. 2011, Ewers et al. 2013). Depending on the relative contribution of each component to total $\beta$-diversity, different conservation strategies are needed to preserve regional species diversity (Angeler 2013). For instance, a dominance of the nestedness component of dissimilarity means low complementarity among sites and implies the need of prioritizing sites with high $\alpha$-diversity, while a predominance of the replacement component would require the conservation of multiple sites at the landscape scale. In order to protect regional biodiversity, it is therefore crucial to understand what ecological phenomena shape each component of $\beta$-diversity (i.e., nestedness and replacement). The latter allows one to fully link scale dependent processes to biodiversity patterns (Leprieur et al. 2011, Angeler 2013) and hence effectively inform management practices.

The metacommunity approach provides a strong conceptual framework to investigate the extent to which local environmental conditions interact with dispersal in determining biodiversity patterns at local and regional spatial scales (Shurin 2001, Leibold et al. 2004, Cottenie 2005, Logue et al. 2011). Traditionally, however, studies investigating the drivers of replacement and nestedness have focused at a biogeographical scale, and most metacommunity studies have not distinguished the two components of $\beta$-diversity (but see Brendonck et al. 2015, Tonkin 
et al. 2016 for some exceptions). At the biogeographical scale (i.e., assuming a history of very low or no dispersal among regions), spatial isolation mainly results in species replacements (i.e., spatial turnover) due to the long-term evolutionary processes of speciation and extinction, which creates differences among regional species pools (McKnight et al. 2007, Melo et al. 2009, Leprieur et al. 2011). Conversely, spatial isolation has been suggested to increase the relative importance of nestedness and reduce the importance of species replacement (i.e., spatial turnover) at the metacommunity scale (Henriques-Silva et al. 2013, Bender et al. 2016). Yet, it is not entirely clear if this relative increase in nestedness is solely driven by spatial isolation or rather depends on the interaction between isolation and environmental constraints (Henriques-Silva et al. 2013). Although dispersal has been suggested to play a central role in determining $\beta$-diversity and its components across spatial scales (e.g., Hortal et al. 2011, Leprieur et al. 2011, Tonkin et al. 2016), the majority of observational studies use spatial variables as an indirect proxy of dispersal rates rather than linking the patterns of diversity directly to actual dispersal rates. This has intrinsic limitations, because pure spatial signals on $\beta$-diversity patterns in observational studies can result either from dispersal limitation or from unmeasured environmental variables that are spatially structured (Peres-Neto et al. 2006, Dray et al. 2012). While it is often difficult to measure dispersal rates in the field, dispersal can be controlled in experiments. Experiments with metacommunities therefore allow a more direct assessment of the potential role of dispersal in shaping diversity patterns, while additional factors can be controlled for (Logue et al. 2011, Verreydt et al. 2012).

The main goal of this study is to experimentally investigate potential drivers of the nestedness and replacement components of $\beta$-diversity in metacommunities. We expect that decreasing dispersal rates, for instance due to increasing fragmentation, will result in an increase of the nestedness component of $\beta$-diversity in environmentally heterogeneous landscapes (Fig. 1a; Henriques-Silva et al. 2013, Bender et al. 2016). In more connected metacommunities the contribution of the nestedness component may be reduced because dispersal rescues species from local extinction (Hanski 1998). At the same time, high dispersal allows species to track suitable environmental conditions at the regional scale (Cottenie and De Meester 2004, Leibold et al. 2004), increasing species replacement via species sorting. Therefore, we anticipate a decrease in the relative contribution of the nestedness component and an increase in the replacement component to total $\beta$-diversity with increasing dispersal rates in environmentally heterogeneous landscapes (Fig. 1a). Only under extremely high dispersal species replacement will decrease due to mass effects (Mouquet and Loreau 2003). Ultimately, such a shift from the nestedness to the replacement component may stabilize total $\beta$-diversity along a broad range of dispersal rates and landscape connectivity scenarios (Fig. 1a).
We expect distinct patterns of $\beta$-diversity derived from nestedness and replacement along dispersal gradients in environmentally homogeneous landscapes. We anticipate that the nestedness component in homogeneous landscapes will be low and barely change along the dispersal gradient for two reasons. First, although we expect some random species extinctions in homogeneous, unconnected landscapes, nestedness is all about non-random, directional species losses (Legendre 2014). Secondly, increasing dispersal in environmentally homogeneous landscapes can result in rapid homogenization of metacommunities (Declerck et al. 2012), which reduces differences in $\alpha$-diversity and maintains the nestdeness component of $\beta$-diversity low across the dispersal gradient (Fig. 1b). In contrast, we expect that in the absence of dispersal, a certain degree of species replacement will be generated by stochastic drift (Fig. 1b; Logue et al. 2011). Increasing dispersal will lead to convergence in species composition due to homogenization (Fig. 1b; Mouquet and Loreau 2003).

We here investigate the independent and interacting effects of dispersal and environmental heterogeneity on the relative contribution of the nestedness and replacement components to $\beta$-diversity of zooplankton metacommunities. Eutrophication is an important anthropogenic pressure worldwide and has been shown to strongly reduce biodiversity in multiple organism groups in shallow lakes and ponds (Scheffer 2004, Declerck et al. 2005, Kruk et al. 2009). Eutrophication gradients may result in species losses and generate community dissimilarity via nestedness. Alternatively, eutrophication gradients may also drive species replacement if species differ in their tolerances to eutrophication (Declerck et al. 2007). Yet, it is largely unknown whether and how varying dispersal rates would alter the relative importance of the nestedness and replacement components resulting from eutrophication. We hypothesize that the influence of eutrophication on the nestedness and replacement components of $\beta$-diversity changes depending on dispersal rates and environmental heterogeneity following the patterns depicted in the conceptual Fig. 1. To test this, we reanalyzed data from a mesocosm experiment in which nutrient concentrations and dispersal rates were manipulated independently (Verreydt et al. 2012). Specifically, we test three key ideas: (1) eutrophication leads to directional species losses due to differences in susceptibility to eutrophication, which enhances the importance of the nestedness component of $\beta$-diversity in less connected, heterogeneous landscapes; (2) increasing dispersal enhances the importance of the replacement and reduces the importance of the nestedness component in heterogeneous landscapes; (3) increasing dispersal reduces the importance of replacement and total $\beta$-diversity in homogeneous landscapes, whereas the nestedness component remains low and unchanged across the dispersal levels. We tested our predictions by focusing on both the metacommunity of mesocosms exposed to different nutrient levels (heterogeneous landscape) and exposed to the same nutrient level (homogeneous landscape) across dispersal levels. 


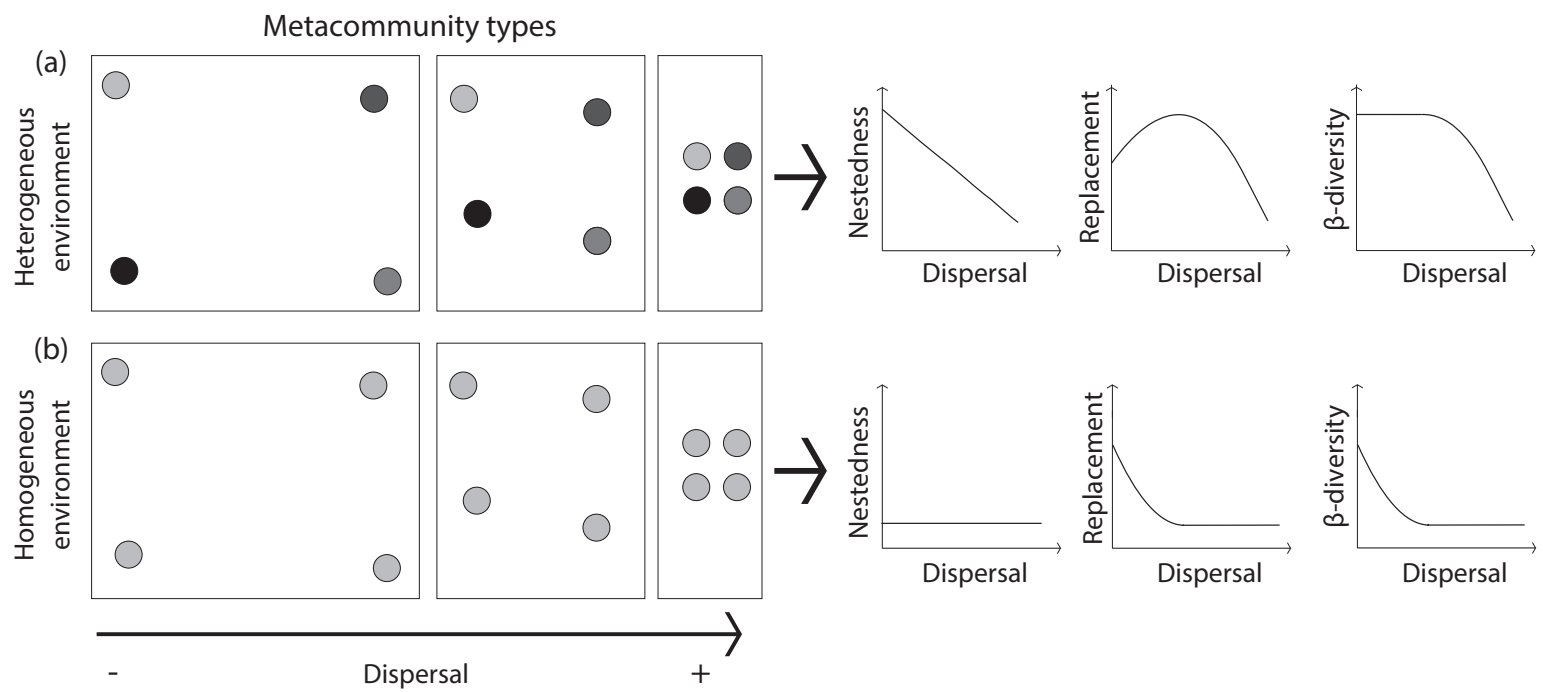

FIG. 1. Conceptual scheme representing the expected relationships between environmental heterogeneity, dispersal rates, and community dissimilarity derived from nestedness and replacement in metacommunities. In a landscape characterized by environmental heterogeneity (e.g., represented by different colors in panel a), the nestedness component is expected to decrease with increasing dispersal because species are rescued from extinction, whereas the replacement component is enhanced with increasing dispersal rates up until a point where mass-effects start to homogenize species composition. The result is that $\beta$-diversity remains high, but has different proportions of the nestedness vs. the turnover components until landscape connectivity is so high that mass effects come into play. In homogeneous landscapes (represented by similar colors of the circles in panel b), the nestdness component may not significantly change along the dispersal gradient (see text for details). Strong dispersal limitation in homogeneous landscapes can cause a pattern of species replacement due to stochastic variation, while increasing dispersal in homogenous environments rapidly reduces replacement due to homogenization. We note that the responses to environmental heterogeneity as shown in the figure only apply to gradients of environmental stressors that exclude species and can lead to nestedness (e.g., eutrophication, pollution, acidification). The patterns depicted here are expected to be general for metacommunities exposed to environmental pressures and that become increasingly fragmented and isolated. Historically unconnected landscapes may show different patterns (e.g., at the biogeographic scale; see Leprieur et al. 2011).

\section{Materials And Methods}

\section{Experimental design and sampling}

We used data from a cross-factorial pond mesocosm experiment $(n=96$; Verreydt et al. 2012) in which three levels of dispersal and two levels of nutrient concentrations were manipulated (see details below). At the start of the experiment (23 May 2006), 96 plastic containers were filled (volume: $200 \mathrm{~L}$ ) with a mixture of $120 \mathrm{~L}$ distilled water and $60 \mathrm{~L}$ tap water. The nutrient treatments were established $5 \mathrm{~d}$ after filling the mesocosms through addition of phosphate $\left(\mathrm{KH}_{2} \mathrm{PO}_{4}\right)$ and nitrogen $\left(\mathrm{NaNO}_{3}\right)$. Initial nutrient additions were equivalent to $1,000 \mu \mathrm{g} \mathrm{P} / \mathrm{L}$ and $16,000 \mu \mathrm{g} \mathrm{N} / \mathrm{L}$ in the high nutrient (HNUT) mesocosms and $10 \mu \mathrm{g} \mathrm{P} / \mathrm{L}$ and $160 \mu \mathrm{g} \mathrm{N} / \mathrm{L}$ In the low nutrient (LNUT) mesocosms. A tenth of these concentrations were added weekly to maintain nutrient concentrations throughout the experiment. Phytoplankton and bacterioplankton were inoculated just after the nutrient addition took place (i.e., fifth day). After we observed a consistent difference in phytoplankton biomass between LNUT and HNUT treatments (day 32), we inoculated zooplankton. Zooplankton communities from 16 different shallow lakes in Belgium were used to inoculate blocks of six mesocosms per lake $(6 \times 16=96$ mesocosms; see Verreydt et al. 2012 and supplementary information therein for more details about the lakes and communities).

Within each set of six mesocosms, we created two levels of nutrient addition (low and high) and three levels of dispersal intensity (no dispersal, low dispersal and high dispersal). In each block (i.e., zooplankton source), the no dispersal (NDISP) and low dispersal (LDISP) mesocosms were inoculated with zooplankton originating from one single lake, whereas the high dispersal (HDISP) mesocosms were initially inoculated with $80 \%$ of zooplankton from the corresponding single lake and a plankton mixture containing zooplankton from all 16 lakes (i.e., 20\%). All dispersal levels started with the same regional species pool (see also Appendix S1: Fig. S4), meaning that inocula from all 16 lakes were present when combining all mesocosms of a given dispersal level $(n=32)$. In contrast to the other two dispersal treatments, however, in the HDISP treatment we created a situation in which all habitat patches were already connected at the start of the experiment, so that each species present in the regional species pool was given a chance to enter each local community from the beginning. The mesocosms of the NDISP treatment were kept isolated from the rest of the experimental metacommunity throughout the entire duration of the experiment. The other two dispersal levels 
were achieved by manually exchanging water among mesocosms. For this, water was collected from all mesocosms of the respective dispersal level $(n=32)$ and the pooled volume was redistributed in equal parts over the same mesocosms again. We exchanged $40 \mathrm{~mL}$ per mesocosm for the LDISP whereas the high dispersal level (HDISP) was achieved by exchanging $2 \mathrm{~L}$ volumes at weekly intervals. Because of our approach to simulate dispersal, dispersal rates of particular species were a function of their relative abundances within local communities. This likely reflects natural dispersal mechanisms in metacommunities of passive dispersers.

The zooplankton community in each mesocosm was sampled at days 86 and 87 of the experiment using a Schindler Patalas (volume: $12 \mathrm{~L}$; mesh size: $30 \mu \mathrm{m}$ ). Samples were preserved with acid lugol solution and individuals were counted and identified in the laboratory. Cladocerans were identified to species level, while copepods were only grouped into cyclopoids and calanoids. A minimum of 300 individuals from each sample was counted. For more details on the experimental design and protocol we refer to (Verreydt et al. 2012).

\section{Statistical analyses}

Effects of nutrient addition and dispersal on $\alpha$-diversity.We used two-way analysis of variance to test for the effects of dispersal (3 levels) and nutrient addition (2 levels) on logarithmic transformed species richness and Shannon entropy (exponential of the Shannon index; Jost 2006), two measures of $\alpha$-diversity.

Calculation of total $\beta$-diversity and its components.Bray-Curtis dissimilarity coefficients based on species abundances were calculated for pairs of experimental communities and used as a measure of $\beta$-diversity. Next, we partitioned $\beta$-diversity into its two components, nestedness and replacement, using the bray part function from the "betapart" statistical package (Baselga and Orme 2012) in R ( $\mathrm{R}$ Core Team 2014). The output of this partitioning approach consists of three distance matrices representing: (1) $\beta$-diversity-i.e., Bray-Curtis; (2) the nestedness component of $\beta$-diversity; and (3) the replacement component of $\beta$-diversity. It is important to note that the nestedness component of $\beta$-diversity as calculated in this study is conceptually similar but yet different from the nestedness that is derived from multisite analyses (Leibold and Mikkelson 2002; for a detailed explanation about the conceptual differences between "nestedness" and "the nestedness component of $\beta$-diversity," Baselga 2012).

We decided to account for species abundances because quantitative data has been shown to provide more useful information on the mechanisms shaping diversity patterns within and among communities (Ulrich and Gotelli 2010, Baselga 2013). The "nestedness component of dissimilarity" in this study will therefore be $>0$ in two situations: (1) when some species disappear from (or colonize) one site but not the other and/or (2) when some species become consistently less (or more) abundant in one site than in the other (Baselga 2013). The previously mentioned situations can happen, for example, due to a change in the environment that directionally affects species abundances or extinctions. Likewise, the replacement component of dissimilarity will be $>0$ when: (1) some species completely replace each other and/or (2) when they change in relative abundances between sites (Baselga 2013). We removed one mesocosm (belonging to the HNUT-NODISP treatment) from the analysis because no species was present in this mesocosm at the end of the experiment.

Effects of nutrient addition and dispersal on $\beta$-diversity and its components. - To test the independent and interacting effects of nutrient addition and dispersal on total $\beta$-diversity and its two components (i.e., nestedness and replacement) we performed multi-factorial PERMANOVA (Anderson and Walsh 2013) using the function "adonis" from the package "vegan" (Oksanen et al. 2016) in R. Multi-factorial PERMANOVA determines whether the level of dissimilarity among mesocosms from different treatments is higher than what would be expected by chance. Multi-factorial PERMANOVA generates tests of significance based on Monte Carlo randomizations, and also estimates $R$-square $\left(R^{2}\right)$ values quantifying the explanatory power of the investigated factors (dispersal and nutrient levels in this study). We used these $R^{2}$ values to assess the relative magnitude of the dispersal and nutrient addition effects on total $\beta$-diversity and its two components. We ran PERMANOVA analysis using dispersal and nutrient levels as factors and as response variables the three distance matrices. Through this analysis, it was also possible to test for an interaction effect between dispersal and nutrients.

Effect of nutrient addition on $\beta$-diversity and its components within each dispersal level.-In a second step, we carried out PERMANOVAs to evaluate the effect of the nutrient gradient (HNUT vs. LNUT mesocosms) on total $\beta$-diversity as well as on the nestedness and replacement components within each dispersal level separately. For this, we calculated total Bray-Curtis, the nestedness and replacement components within each dispersal treatment across the nutrient levels in the same way as described before. We then used those three distance matrices as response variables (one at a time) and nutrient level as a factor.

Effect of dispersal on $\beta$-diversity and its components within each nutrient level.-In order to test whether the level of nestedness and replacement within each nutrient treatment (i.e., mimicking homogeneous landscapes) differ as a function of dispersal rates, we used PERMDISP. This analysis tests whether within group dissimilarity 
in species composition differs among groups (Anderson et al. 2006). To run PERMDISP we used the function "betadisper" in the "vegan" package using as response variables the nestedness and replacement distance matrices and dispersal as a factor. We then used the function "TukeyHSD" to test for significant pair-wise differences between dispersal levels.

\section{RESULTS}

Effects of nutrient addition and dispersal on $\alpha$-diversity

Analyses of variance revealed that nutrient addition had a strong negative effect on species richness $(F=100.783, P<0.001)$ and Shannon entropy $(F=47.671$, $P<0.001)$, whereas dispersal had profound positive effects on species richness $(F=30.013, P<0.001)$ and Shannon entropy $(F=5.351, P=0.006$; see also Appendix $\mathrm{S} 1)$. We found no indication for an interaction effect between dispersal and nutrient addition for species richness $(F=2.662, P=0.075)$ or for Shannon entropy $(F=0.397, P=0.673)$.

\section{Effects of nutrient addition and dispersal on $\beta$-diversity and its components}

Multi-factorial PERMANOVA revealed that the effect of nutrient addition ( ${ }_{\text {adj }} R^{2}=0.123, P=0.001$ ) was twice as strong as the effect of dispersal ( $\left.{ }_{\text {adj }} R^{2}=0.063, P=0.001\right)$ in affecting $\beta$-diversity (Fig. 2a). There was also a significant interaction between dispersal and nutrient addition on $\beta$-diversity ( ${ }_{\mathrm{adj}} R^{2}=0.051, P=0.001$; Fig. $\left.2 \mathrm{a}\right)$. When considering each component of $\beta$-diversity separately, we found a strong effect of dispersal $\left(\mathrm{adj}^{2} R^{2}=0.149, P=0.001\right)$ and a significant interaction between nutrient addition and dispersal rates on the nestedness component $\left({ }_{\text {adj }} R^{2}=0.061, P=0.042\right.$; Fig. 2 b). We found no effect of nutrient addition on the nestedness component (adj $\left.R^{2}=-0.010, P=0.907\right)$. In contrast, nutrient addition had a strong effect on the replacement component (adj $R^{2}=0.207, P=0.001$; Fig. $2 \mathrm{c}$ ), and there was a significant interaction between nutrient addition and dispersal on the replacement component $\left({ }_{\mathrm{adj}} R^{2}=0.056, P=0.012\right)$.
We found no significant effect of dispersal on the replacement component ( $\left.{ }_{\mathrm{adj}} R^{2}=0.038, P=0.055\right)$.

\section{Effect of nutrient addition on $\beta$-diversity and its components within each dispersal level}

One-way PERMANOVAs testing for the effect of nutrient concentration in each dispersal treatment separately revealed a significant effect of nutrient addition on the nestedness component but only in the unconnected mesocosms (NODISP; Fig. 3). In contrast, nutrient addition had no significant effect on the replacement component in the NODISP mesocosms, but was highly significant in the LODISP and, especially, in the HIDISP treatments (Fig. 3). In the absence of dispersal, the nutrient addition treatment thus generated $\beta$-diversity solely via a nestedness pattern. In contrast, in mesocosms with low or high dispersal, $\beta$-diversity associated with the nutrient addition treatment was entirely structured according to a replacement pattern. The effect of nutrient heterogeneity on $\beta$-diversity ranged from $9 \%$ in the NODISP treatment $\left(R^{2}=0.091, P<0.005\right)$ to $11 \%$ in the LODISP $\left(R^{2}=0.108, P<0.001\right)$ to $39 \%$ in the HIDISP treatment $\left(R^{2}=0.393, P<0.001\right)$.

\section{Effect of dispersal on $\beta$-diversity and its components within each nutrient level}

PERMDISP revealed a highly significant difference in replacement within each nutrient treatment as a function of dispersal $(P<0.001$ for both HNUT and LNUT treatments). Pair-wise comparisons revealed that the level of replacement within each nutrient treatment was highly significant between dispersal levels for both nutrient treatments, especially between the NODISP and HIDISP treatments (Appendix S1: Table S3). In contrast, we found no significant differences in the nestedness component within each nutrient treatment as a function of dispersal (Appendix S1: Table S3). Ternary graphs revealed a sharp decrease in replacement and an increase in similarity with increasing dispersal rates within each nutrient treatment (see Appendix S1: Fig. S2). Specifically, average replacement decreased from 0.63 in NODISP mesocosms (a) $\beta$-diversity

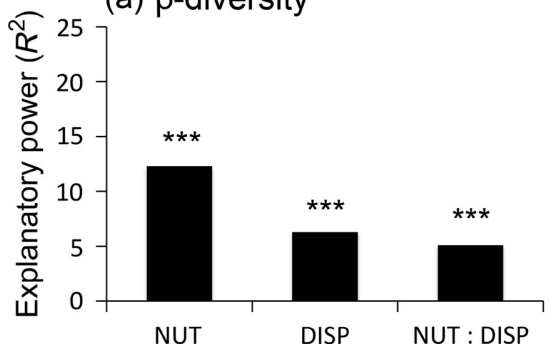

(b) Nestedness

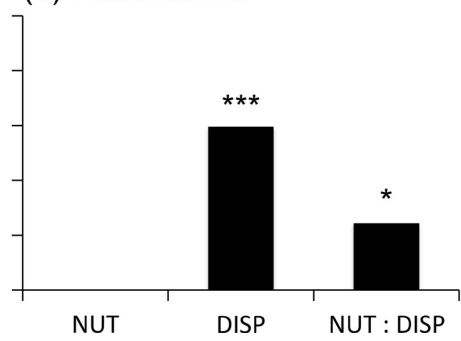

(c) Replacement

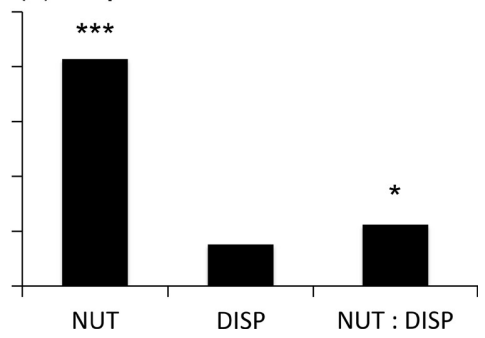

FIG. 2. Total amount of explanatory power $\left(R^{2}\right.$; scaled to 100$)$ provided by the nutrient treatment (NUT), the dispersal treatment (DISP) and their interaction (NUT : DISP) on $\beta$-diversity (a) and its components nestedness (b) and replacement (c). Effects of the treatments on $\beta$-diversity and its components were calculated based on PERMANOVA (see Methods for details). $* * * P<0.001 ; * P<0.05$ 


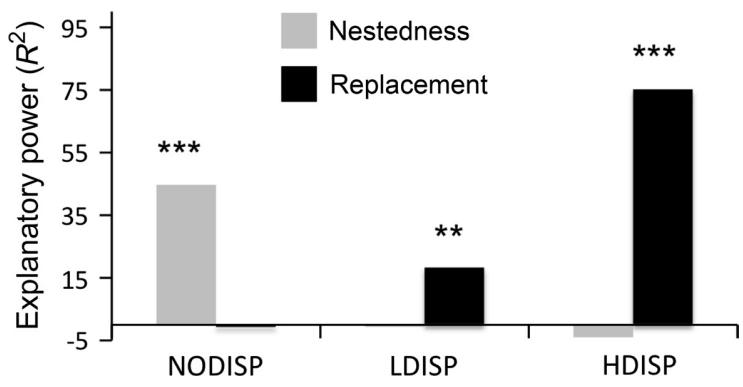

FIG. 3. Total amount of explanatory power $\left(R^{2}\right.$; scaled to $100)$ provided by the nutrient treatment on the nestedness component (grey bars) and on the replacement component (black bars) for each dispersal level separately. NODISP stands for the no dispersal treatment, LDISP refers to the low dispersal treatment, and HDISP to the high dispersal treatment. *** $P<0.001 ; * * P<0.005$.

to 0.15 in HIDISP mesocosms exposed to high nutrient levels (HNUT). Similarly, average replacement decreased from 0.62 in NODISP mesocosms to 0.28 in HIDISP mesocosms with low nutrients (LNUT). There was a slight increase in the relative importance of the nestedness component with increasing dispersal rates, albeit this was not significant (see also Appendix S1: Fig. S2 and Table S3 for all pair-wise comparisons between treatments).

\section{Discussion}

Eutrophication due to nutrient loading and reduced dispersal owing to habitat fragmentation are global scale pressures that are increasingly affecting biodiversity patterns at multiple spatial scales (Kruk et al. 2009, Jeppesen et al. 2010, Hooftman et al. 2015). The results of our crossfactorial mesocosm experiment clearly demonstrate that nutrient enrichment strongly reduced local species richness and diversity, especially in isolated zooplankton communities. Consequently, the nestedness component of $\beta$-diversity was dominant across nutrient levels in unconnected mesocosms. In more connected communities, however, the loss of species due to nutrient additions was counteracted by dispersal, and this shifted the pattern of dissimilarity derived from nestedness to a pattern of dissimilarity derived from species replacement. Thus, the interaction of nutrient addition and dispersal affected the components of $\beta$-diversity in divergent ways. This important interaction would never have been appreciated if the analyses had solely focused on total $\beta$-diversity patterns (Figs. 1, 2a). In addition, we observed contrasting patterns for mesocosms belonging to the same nutrient levels (mimicking an environmentally homogeneous landscape) when exposed to different levels of dispersal. Here, we observed a sharp decrease in both $\beta$-diversity and species replacement with increasing dispersal levels, whereas the contribution of the nestedness component did not significantly change as a function of dispersal (Appendix S1: Table S1). The contribution of the nestedness and replacement components to community dissimilarity across the dispersal levels for heterogeneous landscapes are fully in line with our predictions as outlined in Fig. 1 if we assume that even the high dispersal treatment did not result in mass effects (which is in agreement with the pattern of strong species sorting reported for zooplankton in this dispersal treatment by Verreydt et al. [2012]). Also the patterns of $\beta$-diversity as well as that of the nestedness and replacement components for homogeneous landscapes are largely in line with our predictions.

In the absence of dispersal, the negative effect of nutrient addition on local species richness along with a nested structure indicates that nutrient enrichment creates unsuitable conditions for a subset of zooplankton species. The observed pattern of directional species losses extends the conclusions of several field and experimental studies, which have demonstrated the negative effects of eutrophication on species diversity for many organism groups, including zooplankton (Dodson et al. 2000, Declerck et al. 2007, 2011, Kruk et al. 2009). Nutrient addition impacted $\alpha$-diversity negatively across all dispersal treatments (Appendix S1: Fig. S1). However, this effect was entirely compensated by high dispersal rates (Appendix S1: Fig. S1) resulting in a reduction of the nestedness component of $\beta$-diversity (Fig. 3). This provides evidence for dispersal-mediated rescue effects in our mesocosm experiment (Hanski 1998; see also Appendix S1: Fig. S4). Increased dispersal also increased the importance of species replacement to $\beta$-diversity across the nutrient treatment. Specifically, dispersal fuelled species sorting by allowing species to spatially track suitable environmental conditions at the metacommunity scale, resulting in high complementarity in species composition (Cottenie and De Meester 2004). Previous studies have similarly demonstrated that immigration from the regional species pool has the power to counterbalance the detrimental effects of disturbance, such as high predation pressure, on zooplankton $\alpha$-diversity and can enhance $\beta$-diversity (Shurin 2001, Howeth and Leibold 2010). We also found that $\beta$-diversity is enhanced in the presence of high dispersal rates across the nutrient levels. Through separating $\beta$-diversity into its nestedness and replacement components, however, we show that dispersal limitation and high dispersal both generate $\beta$-diversity in heterogeneous landscapes, but via completely different mechanisms. Specifically, high nutrient input results in species losses in unconnected mesocosms, thus generating $\beta$-diversity derived from nestedness. Conversely, high dispersal promotes species sorting across the nutrient levels and generates $\beta$-diversity via species replacement.

Contrary to our results, some observational studies found that increasing spatial connectivity diminished species replacement (i.e., spatial turnover) and increased nestedness in different organism groups, ranging from river fish to amphibians and mammals (McKnight et al. 2007, Melo et al. 2009, Leprieur et al. 2011). A potential explanation for these differences in outcome is spatial and temporal extent, since the studies above have focused at a biogeographic scale while ours focused at a metacommunity scale. At a biogeographic scale, reduced 
landscape connectivity likely promotes long-term evolutionary processes such as speciation and extinction, which might eventually result in large-scale turnover patterns due to differences in regional species pools (Leprieur et al. 2011). Despite previous theoretical studies on nestedness and turnover at the metacommunity scale, only recently have investigators started assessing the relative importance of dispersal and environmental processes on each component of $\beta$-diversity in empirical metacommunities, especially due to the development of new statistical tools (Baselga 2013, Legendre 2014, Baselga and Leprieur 2015). Using a different approach based on paired overlap and matrix filling to quantify nestedness per se, other studies reported patterns consistent with our results (Henriques-Silva et al. 2013, Bender et al. 2016). For instance, Henriques-Silva et al. (2013) found that harsher environmental conditions in combination with spatial isolation increased nestedness and reduced turnover in fish metacommunities. In another example, Bender et al. (2016) found that spatial isolation is an important driver of functional and taxonomic nestedness. Our findings similarly suggest that heterogeneous metacommunities are shaped by ecological dynamics related to habitat filtering, competitive exclusion and dispersal (Shurin 2001, Bello et al. 2013, Spasojevic et al. 2014), which might create community dissimilarity via species replacement among more connected sites and via nestedness among isolated sites along disturbance gradients.

We found strikingly different patterns for $\beta$-diversity when we considered sets of mesocosms with the same rather than different nutrient levels, i.e., mimicking homogeneous landscapes. In homogeneous landscapes, dispersal limitation enhanced species replacement, whereas the nestedness component remained unchanged across the dispersal levels. The pattern of decreasing species replacement with increasing dispersal in homogeneous landscapes is in line with our predictions, and suggests that the same level of (high) dispersal promotes divergence in species composition in heterogeneous landscapes and community convergence in homogeneous landscapes (Leibold et al. 2004, Cottenie 2005, Logue et al. 2011). We observed that the contribution of the nestedness component was overall low and did not significantly change along the dispersal gradient, which is in agreement with our predictions (see Fig. 1 and Appendix S1: Table S3). Changes in $\beta$-diversity as a function of dispersal were thus mainly derived from species replacements and not from nestedness (see also Appendix S1: Fig. S2). Whereas increasing dispersal enhances species complementarity via species sorting in heterogeneous landscapes, decreasing dispersal enhances species complementarity via stochastic drift in homogeneous landscapes. An open question is whether such high species replacement in unconnected and homogeneous landscapes can be translated into functional complementarity. Although we have no data to further test this hypothesis, functional redundancy may be a dominant pattern in homogeneous and unconnected landscapes, meaning that different species with similar traits replace each other among local communities. The latter is likely to happen because species occurring in homogeneous landscapes have to be able to survive to similar environmental filters (Gianuca et al. 2014). Therefore, it is possible that landscape wide environmental homogenization resulting from a common environmental change, such as widespread eutrophication, selects for functionally redundant species, leading to rapid convergence of functional traits in metacommunities.

Increased nutrient loading and reduced dispersal rates owing to fragmentation are amongst the major drivers of species extinctions worldwide and can undermine ecosystem functioning and stability both in terrestrial and aquatic systems (Kruk et al. 2009, Symons and Arnott 2013, Hautier et al. 2015, Hooftman et al. 2015). Our study additionally suggests that nutrient loading and dispersal limitation synergistically enhance each other's effects on $\beta$-diversity. Our results confirm that conserving habitats based only on high levels of $\alpha$-diversity is not sufficient, as it disregards the importance of variation in species composition among habitats (Angeler 2013, Tonkin et al. 2016). Because dispersal limitation interacts with eutrophication to create dissimilarity resulting mainly from nestedness in heterogeneous landscapes, we argue that sufficient habitat connectivity is crucial in allowing species to repopulate suitable sites and also to ensure high complementarity (replacement) among sites (Shurin 2001, Howeth and Leibold 2010). However, the degree of connectivity in natural metacommunities depends not only on the spatial configuration of the habitat patches, but also on the dispersal mode and ability of the focal organism group (De Bie et al. 2012). As a result, different management strategies will often be needed to conserve $\alpha$ - and $\beta$-diversity of different organism groups. Based on our findings, it is conceivable that nestedness resulting from eutrophication will predominate among dispersal-limited groups and turnover will be more important for highly mobile groups within the same landscape (Tonkin et al. 2016). Future studies should, therefore, investigate how nestedness and turnover along disturbance gradients vary among organism groups that represent a range of dispersal abilities and strategies within the same landscape context.

The approach we applied here, experimentally manipulating dispersal and environmental conditions and teasing apart each component of $\beta$-diversity, was key to obtain new insights into how multi-scale assembly processes link local and regional diversity patterns at a metacommunity scale. It is important to note that in our setting dispersal rates always involved heterogeneous sources and were mainly a function of species relative abundances. The no dispersal treatment represents a situation in which a historically connected metacommunity becomes completely isolated, mimicking a fragmentation scenario. Although each dispersal level started with the same regional species pool (i.e., inocula from 16 lakes; see also Appendix S1: Fig. S4), mesocosms belonging to the high dispersal treatment potentially started with higher 
local species richness than mesocosms of the no dispersal and low dispersal treatment. While different dispersal mechanisms could have led to different conclusions (Grainger and Gilbert 2016), the type of dispersal we used in our experiment is likely a common situation in metacommunities involving passively dispersed organisms, such as freshwater zooplankton (Lopes et al. 2016). Although experimental metacommunities represent only a simplified approximation of natural metacommunities, the results from our study are in clear concordance with those of some recent observational studies that used different methods to quantify nestedness and turnover (e.g., Henriques-Silva et al. 2013, Bender et al. 2016). Additionally, our study extends the conclusions of previous observational studies by demonstrating divergent patterns between heterogeneous and homogeneous landscapes across dispersal scenarios, which was possible to accomplish only via experimentation. Therefore, our study provides strong proof of principle of potential mechanisms structuring the nestedness and replacement components of dissimilarity in natural metacommunities, although some caution is recommended when extrapolating experimental findings to natural systems.

In summary, we demonstrate that nutrient enrichment negatively affects local zooplankton diversity and this creates community dissimilarity derived from nestedness in heterogeneous, unconnected metacommunities. However, the balance between the nestedness and replacement components in our experimental metacommunity was completely altered by dispersal, as increasing dispersal reduced nestedness and resulted in high species replacement in heterogeneous landscapes. Furthermore, the patterns of community dissimilarity derived from nestedness and replacement along dispersal gradients are completely altered in homogeneous environments. This also implies that environmental homogenization, which is likely to happen due to a strong common environmental change such as global warming or widespread eutrophication (Moss et al. 2011), will strongly interact with landscape connectivity in its impact on the components of $\beta$-diversity. Taken together with previous analyses of this same dataset (Verreydt et al. 2012), our results indicate that sufficiently high dispersal in heterogeneous landscapes is fundamental because it promotes biodiversity locally and optimizes the match between species and environment in metacommunities. Our study contributes novel insights on the mechanisms shaping $\beta$-diversity by demonstrating how environmental heterogeneity interplays with dispersal to create contrasting patterns of nestedness and replacement in metacommunities.

\section{ACKNOWLEDGMENTS}

We thank Dino Verreydt for kindly sharing the dataset. Tad Dallas, Letícia Quesado, Caroline Souffreau, and an annonymous reviewer provided valuable comments on an earlier version of this manuscript. ATG received a "Science Without Borders" PhD fellowship from CNPq-Brazil (grant number 245629/2012-2). This work was financially supported by KU
Leuven Excellence Center funding PF/2010/07, Belspo IAP project SPEEDY and BiodivERsA project Tippingpond, nationally funded by Belspo.

\section{Literature Cited}

Anderson, M. J., K. E. Ellingsen, and B. H. McArdle. 2006. Multivariate dispersion as a measure of beta diversity. Ecology Letters 9:683-693.

Anderson, M. J., and D. C. I. Walsh. 2013. PERMANOVA, ANOSIM, and the Mantel test in the face of heterogeneous dispersions: What null hypothesis are you testing? Ecological Monographs 83:557-574.

Angeler, D. G. 2013. Revealing a conservation challenge through partitioned long-term beta diversity: increasing turnover and decreasing nestedness of boreal lake metacommunities. Diversity and Distributions 19:772-781.

Baselga, A. 2010. Partitioning the turnover and nestedness components of beta diversity. Global Ecology and Biogeography 19:134-143.

Baselga, A. 2012. The relationship between species replacement, dissimilarity derived from nestedness, and nestedness. Global Ecology and Biogeography 21:1223-1232.

Baselga, A. 2013. Separating the two components of abundancebased dissimilarity: balanced changes in abundance vs. abundance gradients. Methods in Ecology and Evolution 4:552-557.

Baselga, A., and F. Leprieur. 2015. Comparing methods to separate components of beta diversity. Methods in Ecology and Evolution 6:1069-1079.

Baselga, A., and C. D. L. Orme. 2012. betapart: an R package for the study of beta diversity. Methods in Ecology and Evolution 3:808-812.

Bello, F. D., S. Lavorel, S. Lavergne, C. H. Albert, I. Boulangeat, F. Mazel, and W. Thuiller. 2013. Hierarchical effects of environmental filters on the functional structure of plant communities: a case study in the French Alps. Ecography 36:393-402.

Bender, M. G., F. Leprieur, D. Mouillot, M. Kulbicki, V. Parravicini, M. R. Pie, D. R. Barneche, L. G. R. OliveiraSantos, and S. R. Floeter. 2016. Isolation drives taxonomic and functional nestedness in tropical reef fish faunas. Ecography doi: 10.1111/ecog.02293

Brendonck, L., M. Jocqué, K. Tuytens, B. V. Timms, and B. Vanschoenwinkel. 2015. Hydrological stability drives both local and regional diversity patterns in rock pool metacommunities. Oikos 124:741-749.

Cottenie, K. 2005. Integrating environmental and spatial processes in ecological community dynamics. Ecology Letters $8: 1175-1182$

Cottenie, K., and L. De Meester. 2004. Metacommunity structure: synergy of biotic interactions as seletive agents and dispersal as fuel. Ecology 85:114-119.

De Bie, T., et al. 2012. Body size and dispersal mode as key traits determining metacommunity structure of aquatic organisms. Ecology Letters 15:740-747.

Declerck, S. A. J., E. S. Bakker, B. van Lith, A. Kersbergen, and E. van Donk. 2011. Effects of nutrient additions and macrophyte composition on invertebrate community assembly and diversity in experimental ponds. Basic and Applied Ecology 12:466-475.

Declerck, S., M. Vanderstukken, A. Pals, K. Muylaert, and L. D. Meester. 2007. Plankton biodiversity along a gradient of productivity and its mediation by macrophytes. Ecology 88:2199-2210.

Declerck, S. A. J., C. Winter, J. B. Shurin, C. A. Suttle, and B. Matthews. 2012. Effects of patch connectivity and heterogeneity on metacommunity structure of planktonic bacteria and viruses. ISME Journal 7:533-542. 
Declerck, S., et al. 2005. Multi-group biodiversity in shallow lakes along gradients of phosphorus and water plant cover. Ecology 86:1905-1915.

Dodson, S. I., S. E. Arnott, and K. L. Cottingham. 2000. The relationship in lake communities between primary productivity and species richness. Ecology 81:2662-2679.

Dray, S., et al. 2012. Community ecology in the age of multivariate multiscale spatial analysis. Ecological Monographs 82:257-275.

Ewers, R. M., R. K. Didham, W. D. Pearse, V. Lefebvre, I. M. Rosa, J. M. Carreiras, R. M. Lucas, and D. C. Reuman. 2013. Using landscape history to predict biodiversity patterns in fragmented landscapes. Ecology Letters 16:1221-1233.

Gianuca, A. T., R. A. Dias, V. J. Debastiani, and L. D. S. Duarte. 2014. Habitat filtering influences the phylogenetic structure of avian communities across a coastal gradient in southern Brazil. Austral Ecology 39:29-38.

Grainger, T. N., and B. Gilbert. 2016. Dispersal and diversity in experimental metacommunities: linking theory and practice. Oikos 125:1213-1223.

Hanski, I. 1998. Metapopulation dynamics. Nature 396:41-49.

Hautier, Y., D. Tilman, F. Isbell, E. W. Seabloom, E. T. Borer, and P. B. Reich. 2015. Anthropogenic environmental changes affect ecosystem stability via biodiversity. Science 348 : 336-340.

Henriques-Silva, R., Z. Lindo, and P. R. Peres-Neto. 2013. A community of metacommunities: exploring patterns in species distributions across large geographical areas. Ecology 94:627-639.

Hooftman, D. A. P., B. Edwards, and J. M. Bullock. 2015. Reductions in connectivity and habitat quality drive local extinctions in a plant diversity hotspot. Ecography 39:583-592.

Hortal, J., J. A. F. Diniz-Filho, L. M. Bini, M. Á. Rodríguez, A. Baselga, D. Nogués-Bravo, T. F. Rangel, B. A. Hawkins, and J. M. Lobo. 2011. Ice age climate, evolutionary constraints and diversity patterns of European dung beetles. Ecology Letters 14:741-748.

Howeth, J. G., and M. A. Leibold. 2010. Prey dispersal rate affects prey species composition and trait diversity in response to multiple predators in metacommunities. Journal of Animal Ecology 79:1000-1011.

Jeppesen, E., et al. 2010. Chapter 6: Interaction of climate change and eutrophication. Pages 119-151 in M. Kernan, R. W. Battarbee, and B. R. Moss, editors. Climate change impacts on freshwater ecosystems. Wiley-Blackwell, Chichester, UK.

Jost, L. 2006. Entropy and diversity. Oikos 113:363-375.

Kruk, C., L. Rodríguez-Gallego, M. Meerhoff, F. Quintans, G. Lacerot, N. S. Mazzeo, F. Scasso, J. C. Paggi, E. T. H. M. Peeters, and S. Marten. 2009. Determinants of biodiversity in subtropical shallow lakes (Atlantic coast, Uruguay). Freshwater Biology 54:2628-2641.

Legendre, P. 2014. Interpreting the replacement and richness difference components of beta diversity. Global Ecology and Biogeography 23:1324-1334.

Leibold, M. A., and G. M. Mikkelson. 2002. Coherence, species turnover, and boundary clumping: elements of metacommunity structure. Oikos 97:237-250.

Leibold, M. A., et al. 2004. The metacommunity concept: a framework for multi-scale community ecology. Ecology Letters 7:601-613.
Leprieur, F., P. A. Tedesco, B. Hugueny, O. Beauchard, H. H. Dürr, S. Brosse, and T. Oberdorff. 2011. Partitioning global patterns of freshwater fish beta diversity reveals contrasting signatures of past climate changes. Ecology Letters 14: $325-334$.

Logue, J. B., N. Mouquet, H. Peter, and H. Hillebrand. 2011. Empirical approaches to metacommunities: a review and comparison with theory. Trends in Ecology \& Evolution 26: 482-491.

Lopes, P. M., R. Bozelli, L. M. Bini, J. M. Santangelo, and S. A. J. Declerck. 2016. Contributions of airborne dispersal and dormant propagule recruitment to the assembly of rotifer and crustacean zooplankton communities in temporary ponds. Freshwater Biology 61:658-669.

McKnight, M. W., P. S. White, R. I. McDonald, J. F. Lamoreux, W. Sechrest, R. S. Ridgely, and S. N. Stuart. 2007. Putting beta-diversity on the map: broad-scale congruence and coincidence in the extremes. PLoS Biology 5:e272.

Melo, A. S., T. F. L. V. B. Rangel, and J. A. F. Diniz-Filho. 2009. Environmental drivers of beta-diversity patterns in New-World birds and mammals. Ecography 32:226-236.

Moss, B., et al. 2011. Allied attack: climate change and eutrophication. Inland Waters 1:101-105.

Mouquet, N., and M. Loreau. 2003. Community patterns in source-sink metacommunities. American Naturalist 162: 544-557.

Oksanen, J., et al. 2016. vegan: community ecology package. R package version 2.3-5. https://CRAN.R-project.org/package= vegan

Peres-Neto, P. R., P. Legendre, S. Dray, and D. Borcard. 2006. Variation partitioning of species data matrices: estimation and comparison of fractions. Ecology 87:2614-2625.

R Core Team. 2014. R: a language and environment for statistical computing. R Foundation for Statistical Computing, Vienna, Austria. https://www.r-project.org

Scheffer, M. 2004. Ecology of shallow lakes. Springer, Dordrecht, The Netherlands.

Segre, H., R. Ron, N. De Malach, Z. Henkin, M. Mandel, R. Kadmon, and M. Vellend. 2014. Competitive exclusion, beta diversity, and deterministic vs. stochastic drivers of community assembly. Ecology Letters 17:1400-1408.

Shurin, J. B. 2001. Interactive effects of predation and dispersal on zooplankton communities. Ecology 82:3404.

Spasojevic, M. J., S. Copeland, and K. N. Suding. 2014. Using functional diversity patterns to explore metacommunity dynamics: a framework for understanding local and regional influences on community structure. Ecography 37:939-949.

Symons, C. C., and S. E. Arnott. 2013. Regional zooplankton dispersal provides spatial insurance for ecosystem function. Global Change Biology 19:1610-1619.

Tonkin, J. D., S. Stoll, S. C. Jähnig, and P. Haase. 2016. Contrasting metacommunity structure and beta diversity in an aquatic-floodplain system. Oikos 125:686-697.

Ulrich, W., and N. J. Gotelli. 2010. Null model analysis of species associations using abundance data. Ecology 91: 3384-3397.

Verreydt, D., L. De Meester, E. Decaestecker, M.-J. Villena, K. Van Der Gucht, P. Vannormelingen, W. Vyverman, and S. A. J. Declerck. 2012. Dispersal-mediated trophic interactions can generate apparent patterns of dispersal limitation in aquatic metacommunities. Ecology Letters 15:218-226. 